\title{
Trend analysis combined with expert system applied to real-time monitoring of motor running status and warning of abnormal status
}

\author{
Chang-Ming Lin, Sheng-Fuu Lin
}

\begin{abstract}
With the advancement of technology, industrial automation makes power equipment play an indispensable role in the industry. Power equipment is an indispensable source of power for manufacturing. Therefore, if the equipment is under high pressure, high temperature and high load for a long time, if there is an unwarned shutdown, it will cause huge economic losses. Although regular maintenance is used for maintenance work, it can reduce the occurrence of unwarned downtime, but it also consumes a lot of waste of human and material resources. Therefore, if you can know the real-time running status of the power equipment and the possible causes of the fault, early maintenance personnel can perform machine maintenance and troubleshooting to help improve the reliability of the system. Predictive maintenance is a kind of pre-warning and planning correction behavior. Through the analysis of the data, it can detect the initial abnormal reduction of the equipment's additional energy consumption caused by the failure and improve the maintenance efficiency. According to the U.S. Department of Energy, "Operations and Maintenance Best Practices", it can save about 5\% 20\% energy. In this study, the acceleration amplitude is used as the basis for instant detection. Collect the acceleration amplitude of the motor in the normal running state, and count the acceleration amplitude threshold of the motor in the normal running state. Monitor the amplitude of acceleration in real time to observe the running trend of the motor. When the running trend exceeds the normal range, the vibration spectrum characteristics combined with the expert's rule are used to determine the possible abnormal types of the motor.
\end{abstract}

Index Terms-Energy saving, Fault diagnosis, Motor vibration, Percent tolerance pre-control chart, Process.

\section{INTRODUCTION}

Predictive maintenance is based on state maintenance and builds a model that can predict abnormal failures in accordance with production process conditions, thereby achieving pre-warning and planning corrective actions. According to the U.S. Department of Energy, "Operations and Maintenance Best Practices", predicting maintenance and early warning of equipment failure can save about $5 \%$ to $20 \%$ of equipment energy consumption. Many studies have conducted measurement studies on the abnormal energy loss

Chang-Ming Lin, Institute of Electrical and Control Engineering, National Chiao Tung University, 1001 University Road, Hsinchu 30010 +886-3-591-6187.

Sheng-Fuu Lin, Institute of Electrical and Control Engineering, National Chiao Tung University, 1001 University Road, Hsinchu 30010, +886-35712121 ext54365. of rotating equipment-motors. Among them, studies have shown that the energy consumption of a faulty motor increases by about $0.22 \%$ - $4 \%$ relative to a healthy motor. [1] proposed a new framework for change detection in machine monitoring where we introduced the graph model instead of the ARIMA model to capture/represent the statistical dynamics in a monitored machine operational process. [2] proposed an article feature learning is used in the form of a convolutional neural network model, which is an end-to-end machine learning system, and applied the convolutional neural network on this raw data, the network learns transformations on the data that result in better representation of the data for the eventual classification task in the output layer. [3] presented a stand-alone multisensor wireless system for continuous condition monitoring of induction motors. The proposed wireless system provides a low-cost alternative to expensive condition monitoring technology available through dedicated current signature analysis or vibration monitoring equipment. The system employs multiple sensors (acoustic, vibration, and current) mounted on a common wireless platform. The faults of interest are static and dynamic air-gap eccentricity, bearing damage, and their combinations. The Hilbert-Huang transform of vibration data and power spectral density of current and acoustic signals are used as the features in a hierarchical classifier. [4] has investigated the feasibility of detecting bearing faults using a spectrum of a single phase of the stator current of an induction machine. Measured current and vibration spectrums were presented to verify this relationship. While these changes are relatively small when compared to the rest of the current spectrum, they fall at locations that are different from the supply and slot harmonics of the machine. With sufficient spectral resolution, this discrimination makes the bearing harmonics sufficiently distinct for use as effective indicators of rolling-element bearing damage. [5] described two fault-detection schemes for a broken-rotor-bar fault detection with a multiple signature processing and demonstrates that the multiple signature processing is more efficient than a single signature processing. [6] developed a neural network-based incipient fault detector for small- and medium-size induction motors. The neural network-based incipient fault detector avoids the problems associated with traditional incipient fault detection schemes by employing more readily available information such as rotor speed and stator current.

There have been many studies for the detection of motor faults. [7] described a DSP-based architecture for vibration analysis. It allows machine monitoring to be carried out on-line, with a consequent increase in the system and in environmental safety. The use of parametric unfault and fault 
models, estimated by extracting specific characteristics in the vibration spectrum. [8] mentioned that the motor may be disturbed by low-frequency noises during the actual operation, and causes temporary abnormalities in the vibration spectrum of the motor. The author used a neural network to identify the abnormal signal and combined the statistical process control and zone rules together to rule out the temporary fault signal. [9] presented a signal analysis technique for machine health monitoring based on the Hilbert-Huang Transform (HHT). The HHT represented a time-dependent series in a two-dimensional (2-D) time-frequency domain by extracting instantaneous frequency components within the signal through an Empirical Mode Decomposition (EMD) process. Spectrum measurement is commonly used to diagnose the normal state of the motor. [11] presented power spectral density of current and acoustic signals and it used to classify the normal and faulty motors.

This study presents the trend analysis detection algorithm to install an acceleration gauge on the motor, collect the maximum amplitude of the acceleration gauge in the time domain and store it in the database. Calculate the average and standard deviation of the acceleration amplitude. The triple standard deviation range is used as the limit of the maximum amplitude of normal operation. Observe the real-time operation status of the motor, and monitor the current operation status of the equipment by the interval rule and the percent tolerance precontrol chart (PTPCC). Further analyze the frequency spectrum state of the motor, combined with expert knowledge to establish a motor abnormal expert diagnosis system.

\section{BACKGROUND ON MOTOR FAULT}

This paper considers motor malfunctions due to various rotor unbalance, misalignment failure and bearing failure. This section reviews the fault feature of each of these three faults.

\section{A. Rotor Unbalance}

The definition of unbalance is when the axis of inertia of the rotating part and the axis of the rotating axis are not on the same straight line. The reasons for the unbalance of the rotating parts may be the asymmetric shape of the rotating parts, the manufacturing tolerances, the improper assembly and installation, the deformation of the rotating parts or the wear and tear of the rotating parts, or the attachment of the rotating part. The spectrum diagram is shown in Figure 1.

The unbalanced vibration features.

1) Vibration spectrum mainly occurs at $1 \mathrm{X}$ speed.

$$
X=\frac{R P M}{180}
$$

Where RPM is the rotation speed of the motor.

2) The direction of vibration usually occurs in the radial direction.

3) The axial amplitude is very small, much smaller than $1 / 3$ of the radial.

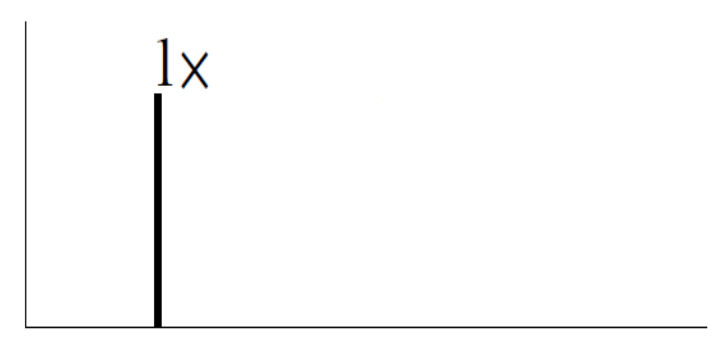

Figure 1. The rotor unbalance spectrum schematic diagram.

\section{B. Misalignment Failure}

Misalignment means that the centerlines of operation of the two devices connected together are not on the same straight line.

The misalignment vibration features.

1. Vibration spectrum mainly occurs at $1 X, 2 X$ or $3 X$ speed. The spectrum diagram is shown in Figure 2.

2. Most of the misalignment is a hybrid misalignment, the vibration direction comes from both radial and axial directions.

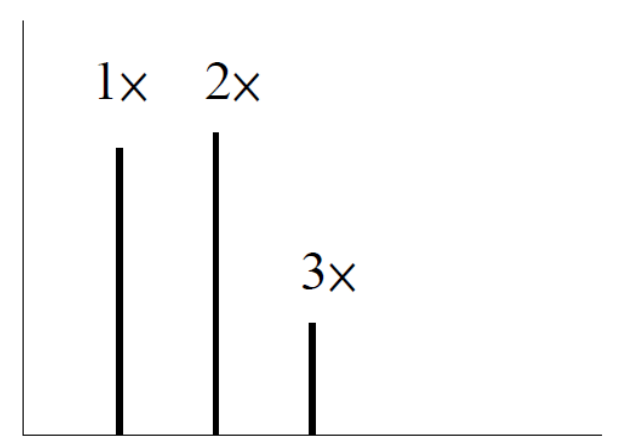

Figure 2. The misalignment failure spectrum schematic diagram.

\section{Bearing Failure}

Bearing failures are the most common failures in induction motors. The major causes of failures are: damages on inner or outer races of the bearing due to thermal or dynamic mechanical stresses. Misalignment and poor bearing fitting can also damage the bearing cage. The other source of bearing fault is lack of lubricant due to thermal or electrical stresses.

Rolling bearings are composed of four main components, including outer ring, inner ring, rolling parts, and retainer. Each of the four components has its own unique frequency.

The unbalanced vibration features are:

1) BPFI is usually speed $\times \mathrm{N} \times 60 \%$.

2) BPFO is usually speed $\times \mathrm{N} \times 40 \%$.

3) FTF is usually the RPM $\times 0.4 \sim 0.6$.

4) BSF is usually 2 4 times the RPM.

Where $\mathrm{N}$ is the amount of bearing rolling parts. BPFI is ball pass frequency inner race. BPFO is ball pass frequency outer race. FTF is fundamental train frequency. BSF is ball spin frequency.

\section{MATERIALS AND METHODS}

\section{A. Experimental Setup}

The detection platform uses vibration detection to diagnose 
rotor unbalance, centering faults and bearing faults. The detection platform used is the PBS-5000 rotary machine rotor disturbance system, as shown in Figure 3. The 4 vibration sensors $(100 \mathrm{mv} / \mathrm{g})$ of the detection platform device output vibration signals at $4-20 \mathrm{~mA}$ to receive vibration signals. The data communication function of this platform is to use the standard interface of the window socket to communicate with the vibration data acquisition module to collect spectrum data and time waveform data. The number of spectrum data points will be determined according to the acquisition bandwidth. For example, if the bandwidth is $1 \mathrm{~K}$ for 801 points, $2 \mathrm{~K}$ for 1601 points, and $10 \mathrm{~K}$ for 3201 points, this plan uses $10 \mathrm{~K}$ 3201 points for testing; the time waveform is determined by the time to be captured, and the communication test is set by collecting 4097 points at a time.

Different types of faults are used to make artifacts with abnormal defects by artificial processing, and then the abnormal artifacts are assembled on the inspection platform to create the fault type of the motor for abnormal diagnosis and analysis. Unbalance is the most common fault in all kinds of rotating machinery. About $70 \%$ of the faults of rotating machinery are related to the unbalance of the rotor. There are many reasons for unbalance, such as unreasonable structural design of rotor, deviation of machining quality, assembly error uneven material, and poor dynamic balance accuracy. In the experiment, the screw is installed on one side of the mass disc to simulate the unbalanced state of the rotor during the operation of the motor. As shown in Figure 4.

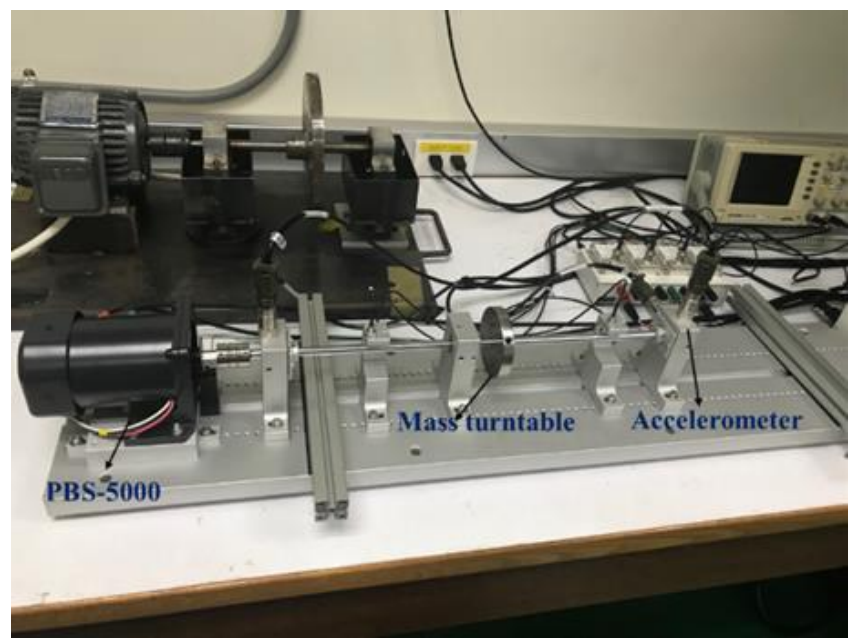

Figure 3. Experimental Setup

Large machinery is usually composed of multiple rotors, and each rotor is connected by a coupling to form a shaft system for transmitting motion and torque. Due to factors such as installation error of the machine, thermal expansion of the working state, deformation of the load and the original unevenness of the machine, etc., the abnormality of the misalignment between the rotors during the operation of the machine occurs. The experimental operation is divided into normal and misaligned faults, and the normal centrifugal rotor is placed in a gasket to create an operating state of misaligned faults, as shown in Figure 5.

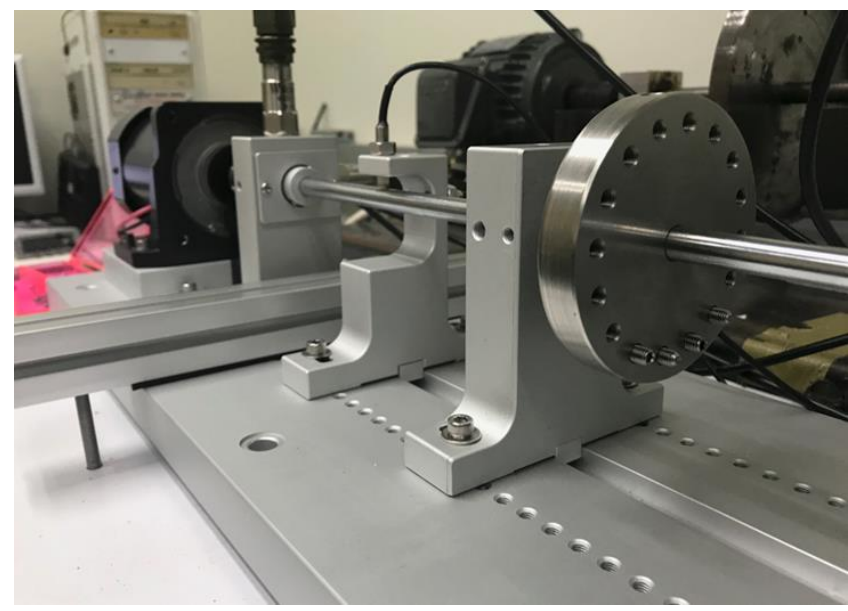

Figure 4. Unbalance Failure Experimental Setup

Rolling bearing failure is a part that is easily damaged in rotating machinery. According to statistics, $30 \%$ of rotating machinery failures are caused by bearing failures, so the quality of bearings has a great impact on rotating machinery. The characteristic frequencies of bearing failures mainly occur in the areas of 1st and 2nd octave with larger amplitude and extremely high octave and lower than 1st octave will also produce higher amplitude than normal. The experimental operation is divided into the state of normal operation and bearing failure, as shown in Figure 6.

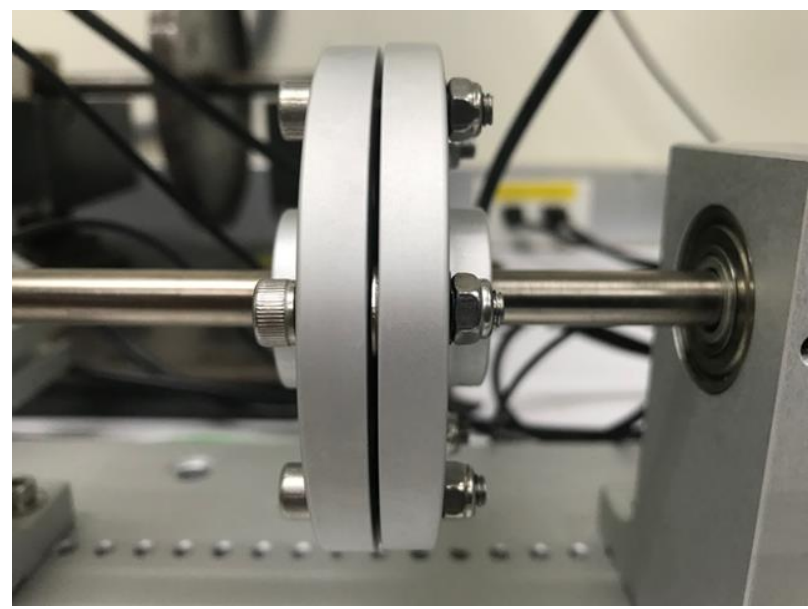

Figure 5. Misalignment Failure Experimental Setup

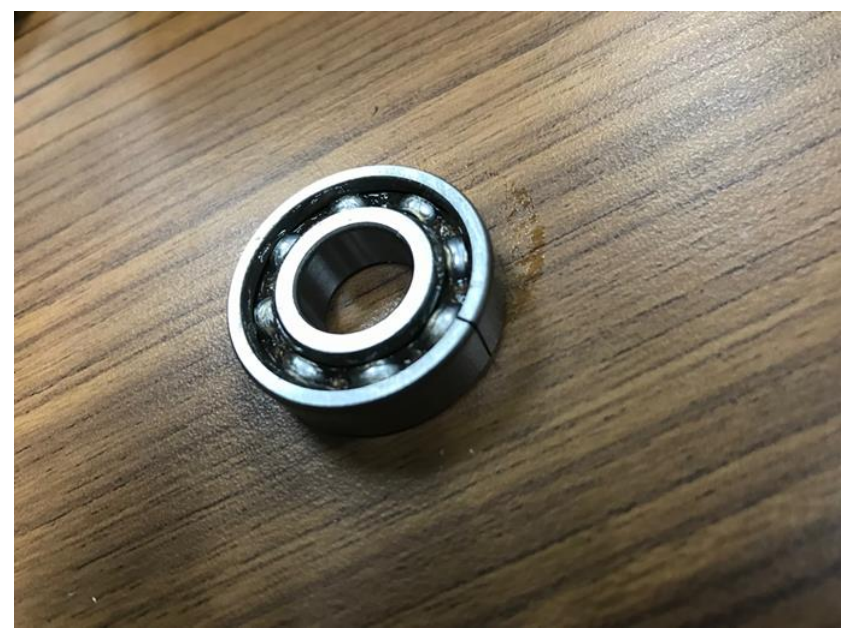

Figure 6. Bearing Failure Experimental Setup 


\section{B. Trend Analysis Detection Algorithm}

Condition monitoring is the detection, recording, analysis and processing of specific characteristic signals during the operation of equipment, and is the basic work of equipment fault diagnosis. The signals collected by the equipment detection are mainly the signals of the equipment in operation (such as vibration, noise, speed, temperature, pressure, flow, etc.) for status monitoring.

Time series refers to a series of values arranged in the order of the occurrence of events. The time domain analysis method establishes a set of mathematical models based on the operating data of the equipment, and uses this model to analyze the changes in the data. This study uses trend analysis detection algorithm to determine the trend range of equipment operation. The value measured during the normal operation of the equipment is set as the initial value, and the difference in the value of the same part of the equipment is instantly compared. Under normal equipment operation, this difference must fall within a reasonable range. The flow chart is shown in Figure 7. Install the acceleration gauge on the motor, collect the maximum amplitude generated by the acceleration gauge on the time domain signal and store the maximum amplitude in the database. When the maximum amplitude has reached a certain number of counts, the average value of the maximum amplitude and the standard deviation of the motor under normal operation can be counted, and the range of three times the standard deviation is set as the limit of normal operation. Use the interval rule and PTPCC to monitor the current running trend of the motor.

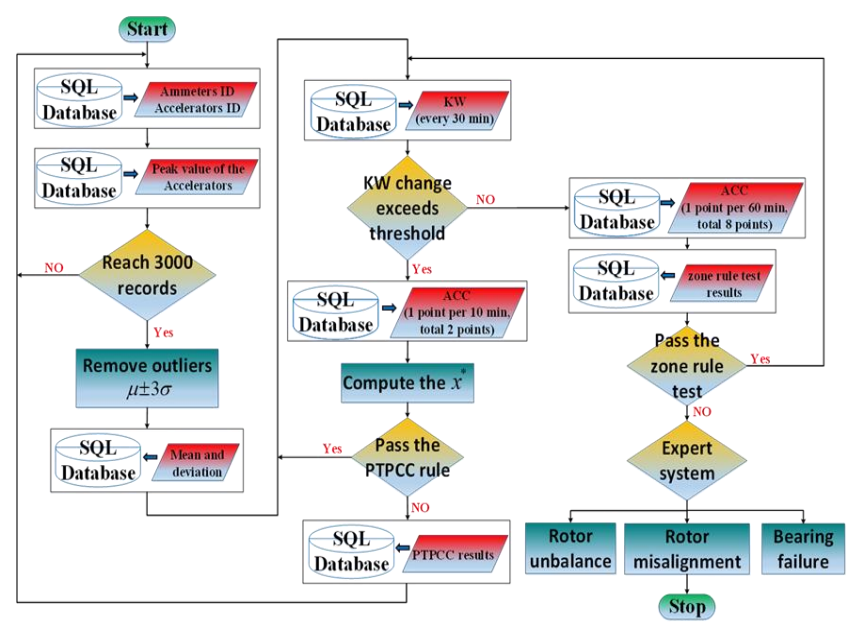

Figure 7. Flowchart of trend analysis detection algorithm

Quality control is used to monitor the quality characteristics of the measured value changes with time. Statistical Process Control (SPC) is a widely used online process monitoring tool. A typical control chart contains a centerline $(\mathrm{CL})$, used to represent the average number of quality characteristics. The control chart also contains two horizontal lines, called upper control limit (UCL) and lower control limit (LCL), as shown in Figure 8. The interval rule is to judge the situation that the monitoring data falls within the control boundary as shown in Figure 9. The standard of the interval rule is shown in Table 1 . If any of the conditions in Table 1 is satisfied, it means that the motor running trend is abnormal.

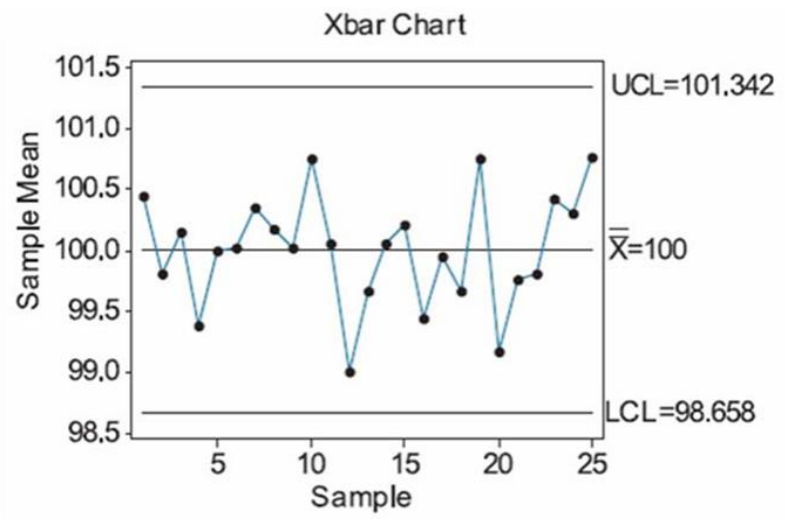

Figure 8. Schematic diagram of SPC

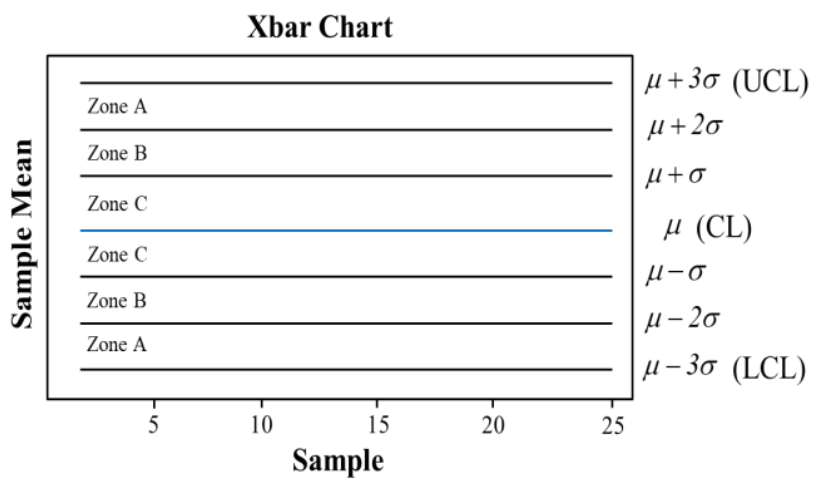

Figure 9. Interval Rule Control Chart

Table 1. Interval rule standard

One point is beyond UCL or LCL

Two of three consecutive points are outside of zone B on the same side of the CL.

Four of five consecutive points are outside of zone $\mathrm{C}$ on the same side of the CL.

Consecutive eight points continuously rising or falling on

the same side of the CL.

The percent tolerance precontrol chart (PTPCC) was proposed by Vermani (2000). This method mainly calculates the tolerance ratio and monitors the process with the concept of pre-transfer to the graph. The calculation formula (2) is as follows:

$$
x^{*}=\frac{2(x-\text { mean })}{(U S L-L S L)}
$$

where $\mathrm{x}$ is the acceleration peak value.

The rule of PTPCC is to take two consecutive samples for inspection. The control chart is shown in Figure 10. The judgment criteria are shown in Figure 11, where $\mathrm{xi}^{*}, \mathrm{i}=1,2$, are from (2) with two sample points x. This study uses PTPCC to determine whether the maximum amplitude limit of acceleration needs to be recalculated, which represents whether the normal operating range of the motor needs to be recalculated. The decision displayed by PTPCC is "R", which means that the observation value of the device changes drastically, and the average value and standard deviation of the maximum acceleration amplitude need to be recalculated. "N" means no recalculation is required. 


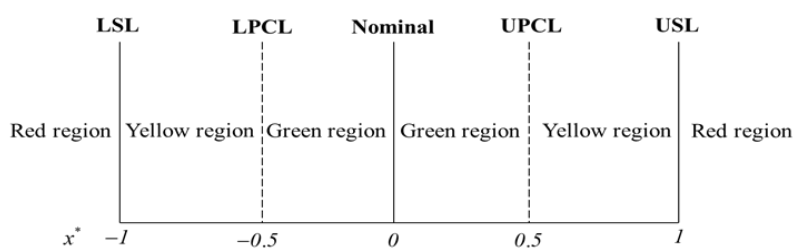

Figure 10. PTPCC control chart

\begin{tabular}{|c|c|c|c|c|c|c|c|c|c|c|c|}
\hline \multirow{2}{*}{$\begin{array}{l}\text { Region } \\
\text { Red }\end{array}$} & \multicolumn{2}{|c|}{$\begin{array}{l}\text { One } x_{i}^{*} \text { in } \\
\text { red region }\end{array}$} & \multicolumn{2}{|c|}{$\begin{array}{l}\text { Two } x_{i}^{*} \text { in } \\
\text { different } \\
\text { yellow regions }\end{array}$} & \multicolumn{2}{|c|}{$\begin{array}{l}\text { Two } x_{i}^{*} \text { in the } \\
\text { same yellow } \\
\text { region }\end{array}$} & \multirow{2}{*}{$\begin{array}{l}\text { Two } \\
x_{i}^{*} \text { in } \\
\text { green } \\
\text { region }\end{array}$} & \multicolumn{4}{|c|}{$\begin{array}{l}\text { One } x_{i}^{*} \text { in green region, and } \\
\text { the other } x_{i}^{*} \text { in yellow region }\end{array}$} \\
\hline & $x_{i}^{*}$ & & & & & & & & & & \\
\hline Yellow & & & $x_{1}^{*}$ & $x_{2}{ }^{*}$ & & $x_{1}^{*} x_{2} z^{*}$ & & & & $x_{1}{ }^{*}$ & $x_{2} *$ \\
\hline Green & & & & & & & $x_{3}^{*} x_{2}^{*}$ & $x_{1} *$ & $x_{2} *$ & $x_{2} *$ & $x_{1} x_{*}$ \\
\hline Yellow & & & $x_{2}{ }^{*}$ & $x_{1}^{*}$ & $x_{1}^{*} x_{2} z_{*}$ & & & $x_{2} *$ & $x_{1} l^{*}$ & & \\
\hline Red & & $x_{i} *_{+}$ & & & & & & & & & \\
\hline Decision & $\mathrm{R}$ & $\mathrm{R}$ & $\mathrm{R}$ & $R$ & $\mathrm{R}$ & $R$ & $\mathrm{~N}$ & $\mathrm{~N}$ & $\mathrm{~N}$ & $\mathrm{~N}$ & $\mathrm{~N}$ \\
\hline
\end{tabular}

Figure 11. PTPCC standard

\section{Expert System}

Expert system is a kind of "knowledge-based" artificial intelligence diagnosis system, which uses a large number of human expert knowledge and reasoning methods to solve complex practical problems. The equipment fault diagnosis expert system is a calculation program compiled from the knowledge, experience, reasoning, and skills of multiple experts in human equipment fault diagnosis. It can be used to analyze and solve complex problems that can only be described in language or thought reasoning.

Different motor failures reflect different vibration signals, and analyze the abnormal types of motors based on these unique failure feature. This research aims at three common types of motor failures, including rotor imbalance, misalignment failures and bearing failures. The vibration spectrum is used to diagnose motor abnormalities according to expert rules. The expert rule is shown in Table 2.

Table 2. Expert rule of motor failure

\begin{tabular}{|l|l|}
\hline Fault type & Fault feature spectrum \\
\hline Rotor imbalance & At $1 X$ \\
\hline Misalignment failure & At $1 X$ and $2 X$. \\
\hline Bearing failure & $\begin{array}{l}\text { At } 1 X, 2 X, \text { and high-power } X \\
\text { spectrum }\end{array}$ \\
\hline
\end{tabular}

\section{DATA ANALYSIS}

This experiment is mainly to measure and collect the acceleration amplitude distribution status of three common abnormal motor faults. The three abnormalities include rotor unbalance, centripetal fault and bearing fault. Taking the data of the motor rotor imbalance as an example, the speed set by the experiment is $2300 \mathrm{rpm}$ as the basis of the analysis speed. The experiment is divided into the motor normal operation experiment and the motor rotor imbalance experiment. The rotor unbalance experiment changes the weight by installing screws on the mass turntable to simulate the unbalanced rotor rotation. The amplitude of the time-domain signal in normal operation falls within \pm 0.15 , and the amplitude of the motor with an unbalanced rotor also increases with severity. As shown in Figure 12 to Figure 15.

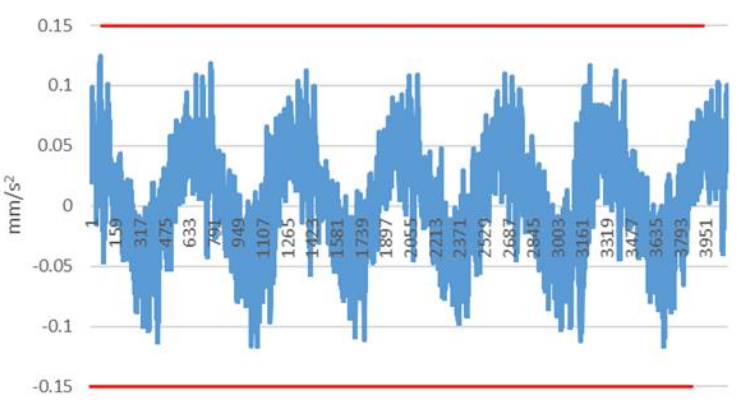

Figure 12. Normal operation time domain signal distribution.

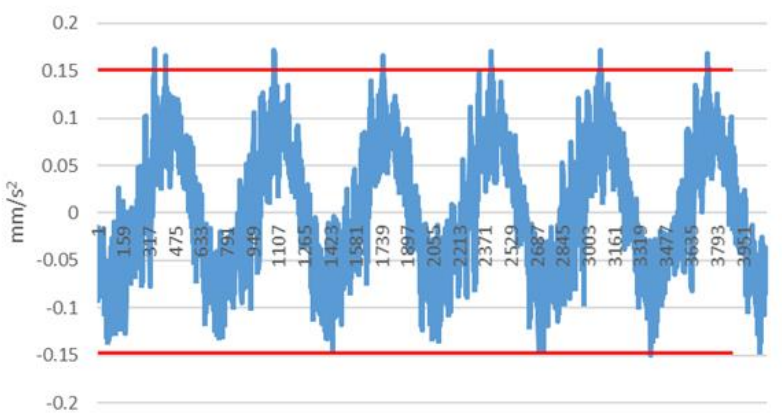

Figure 13. Three screws counterweight operation time domain signal distribution.

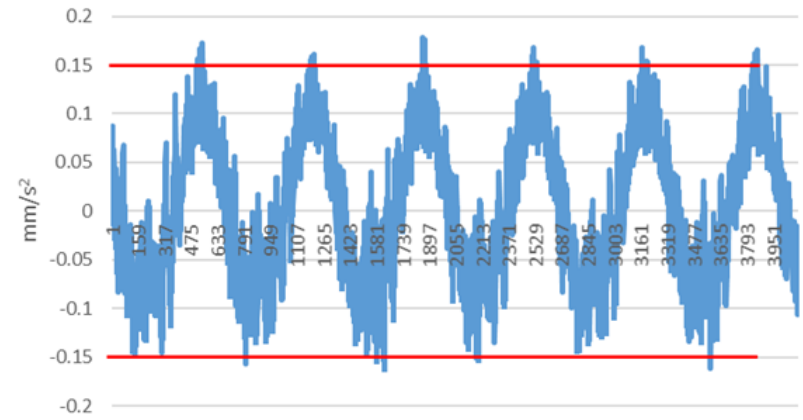

Figure 14. Four screws counterweight operation time domain signal distribution.

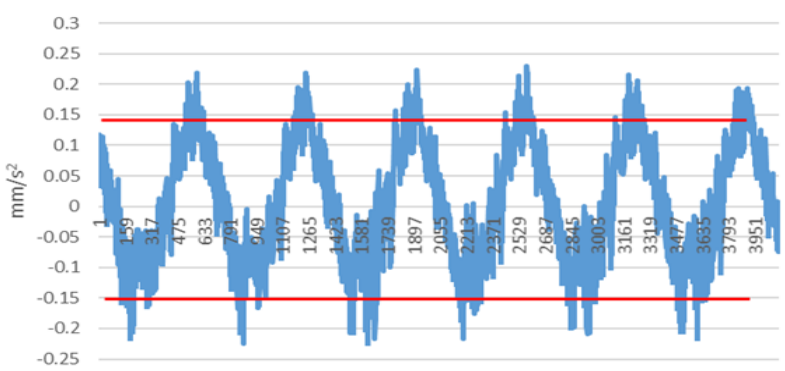

Figure 15. Five screws counterweight operation time domain signal distribution.

The three types of motor failure types, such as rotor unbalance, centripetal failure, and bearing failure, are based on the characteristic frequency of operating failures verified by the expert system. The feature spectrum of various faults in the frequency domain are shown in Figure 16. 
Trend analysis combined with expert system applied to real-time monitoring of motor running status and warning of abnormal status

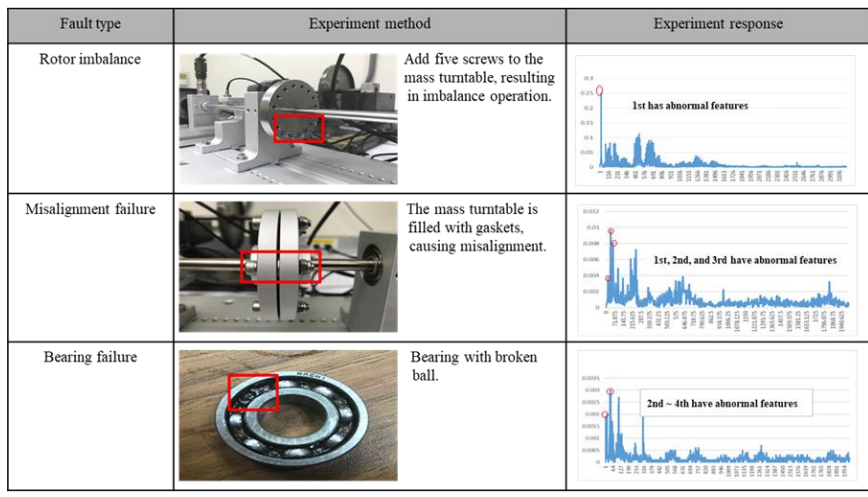

Figure 16. Motor fault abnormal feature spectrum.

The experimental results show that the normal and abnormal motors have a difference of nearly 2.7 times in the amplitude of the $1 \mathrm{X}$ spectrum. The motor with unbalanced rotor has a 1X spectrum higher than that of a normal motor, and the amplitude of the $1 \mathrm{X}$ spectrum will increase with the severity of the abnormality. The same experimental method is applied to the rotor's misalignment and bearing failure to obtain the same acceleration amplitude time-domain signal change trend. The spectrum of different motor failure types can also be diagnosed based on expert penalty.

\section{CONCLUSION}

In this study, an accelerometer is installed on the motor and the acceleration amplitude of the power equipment in normal operation is collected. After accumulating a certain number of acceleration amplitudes, the standard deviation and average value of the acceleration amplitude are calculated. Use the relative standard test to take the acceleration amplitude of normal operation as the initial value, and observe the difference between the acceleration amplitude trend of the power equipment and the initial value. The status is entered into the expert's law diagnosis. On the other hand, the acceleration value undergoes Fourier transform to decompose the signal into simple harmonic components of frequency, and establish the expert law of the characteristic frequency of the fault. When the machine equipment fails, the frequency change is caused by expert rules to help provide the type of possible failure.

\section{REFERENCES}

[1] G. Lu, J. Liu and P. Yan, "Graph-based structural change detection for rotating machinery monitoring," Mechanical Systems and Signal Processing 99 (2018) 73-82

[2] O. Janssens et al., , "Convolutional Neural Network Based Faul Detection for Rotating Machinery," Journal of Sound and Vibration 377(2016) 331-345

[3] S. Wang, V. Sundararajan and E. T. Esfahani, "Multisensor Wireless System for Eccentricity and Bearing Fault Detection in Induction Motors," IEEE/ASME TRANSACTIONS ON MECHATRONICS, VOL. 19, NO. 3, JUNE 2014

[4] R. R. Schoen, T. G. Habetler, F. Kumran and R. G. Bartheld, "Motor Bearing Damage Detection Using Stator Current Monitoring," IEEE TRANSACTIONS ON INDUSTRY APPLICATIONS, VOL. 31, NO. 6, NOVEMBER/DECEMBER 1995

[5] B. Ayhan, M. Y. Chow and M. H. Sing, "Multiple Discriminan Analysis and Neural-Network-Based Monolith and Partition Fault-Detection Schemes for Broken Rotor Bar in Induction Motors," IEEE TRANSACTIONS ON INDUSTRIAL ELECTRONICS, VOL. 53, NO. 4, AUGUST 2006
[6] M. Y. Chow and S. O. Yee, "A Neural Network Approach to Real-Time Condition Monitoring of Induction Motors," IEEE TRANSACTIONS ON INDUSTRIAL ELECTRONICS, VOL. 38, NO. 6, DECEMBER 1991

[7] G. Betta, C. Liguori, A. Paolillo and A. Pietrosanto, "A DSP-Based FFT-Analyzer for the Fault Diagnosis of Rotating Machine Based on Vibration Analysis," IEEE TRANSACTIONS ON INSTRUMENTATION AND MEASUREMENT, VOL. 51, NO. 6, DECEMBER 2002

[8] J. Y. Zheng, Design and Implementation of Intelligent Motor Rotary Fault Diagnosis System Using the Statistical Process Control Technique, Master's thesis, National Taipei University of Technology, 2009

[9] R. Yan and R. X. Gao, "Hilbert-Huang Transform-Based Vibration Signal Analysis for Machine Health Monitoring," IEEE TRANSACTIONS ON INSTRUMENTATION AND MEASUREMENT, VOL. 55, NO. 6, DECEMBER 2006

[10] W. Wang and O. A. Jianu, "A Smart Sensing Unit for Vibration Measurement and Monitoring," IEEE/ASME TRANSACTIONS ON MECHATRONICS, VOL. 15, NO. 1, FEBRUARY 2010

[11] S. A1-Dossary, R. I. R. Hamzah and D. Mba, "Observations of changes in acoustic emission waveform for varying seeded defect sizes in a rolling element bearing," Applied Acoustics 70 (2009) 58-81

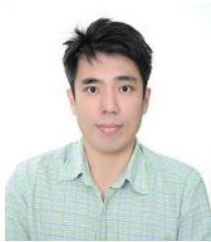
1980. He received the B.C. degrees of mechanical engineering from National Yunlin University of Science \& Technology in 2003 and M.S. degrees in mechanical engineering from Widener University in 2007. Now he is an engineer in Industrial Technology Research Institute (ITRI). His current research interests include data mining and energy-saving control.

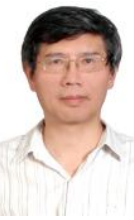

Sheng-Fuu Lin (S'84-M'88-SM'16) was born in Tainan, R.O.C., in 1954. He received the B.C. and M.S. degrees in mathematics from National Taiwan Normal University in 1976 and 1979, respectively, the M.S. degree in computer science from the University of Maryland in 1985, and the Ph.D. degree in electrical engineering from the University of Illinois, Champaign, in 1988.

Since 1988, he has been on the faculty of the Department of Electrical and Control Engineering at National Chiao Tung University, Hsinchu, Taiwan, where he is currently a Professor. His research interests include image processing, pattern recognition, fuzzy theory, and automatic target recognition.

Dr. Lin is a Member of the IEEE, the Chinese Fuzzy System Association and the Chinese Automatic Control Society. 